\title{
Influence of end expiratory pressure on cerebral blood flow in preterm infants
}

\author{
Reinier A. Mullaart*a, Jeroen C.W. Hopman ${ }^{\mathrm{a}}$, Jan J. Rotteveel ${ }^{\mathrm{a}}$, \\ Otto Daniëls ${ }^{\mathrm{a}}$, Gerard B.A. Stoelinga ${ }^{\mathrm{a}}$, Anton F.J. De Haan ${ }^{\mathrm{b}}$, \\ Louis A.A. Kollée ${ }^{\text {a }}$ \\ a Paediatric Divison, University Hospital Nijmegen, Nijmegen, The Netherlands \\ ${ }^{b}$ Department of Medical Statistics, University Hospital Nijmegen, Nijmegen, The Netherlands
}

Received 4 July 1993; accepted 31 October 1994

\begin{abstract}
The effect of interruption of positive end expiratory pressure (PEEP) on cerebral blood flow velocity (CBFV) and CBF fluctuation (CBFF) in the internal carotid arteries and on heart rate, restlessness and wakefulness has been studied in 17 mechanically ventilated neonates with RDS. A decrease in CBFV was found, but no significant change in CBFF. Multiple regression analysis showed that the decrease in CBFV is less pronounced if the PEEP interruption is accompanied by restlessness. It further appeared that the decrease in CBFV is more pronounced if CBFV is high, the ductus arteriosus is patent, or RDS follows a complicated course. These findings indicate that PEEP supports CBF, probably by a decrease in ductal stealing from the brain. Therewith PEEP protects against cerebral hypoperfusion which is one of the major risks in RDS and immaturity. Furthermore, our findings suggest that the decrease in CBF during PEEP interruption is moderated by restlessness and accentuated by brain damage.
\end{abstract}

Keywords: Positive end expiratory pressure; Cerebral blood flow; Respiratory distress syndrome; Patent ductus arteriosus

* Corresponding author, Department of Child Neurology, University Hospital Nijmegen, PO Box 9101, NL-6500 HB, The Netherlands. 


\section{Introduction}

Positive end expiratory pressure (PEEP) is a common treatment modality for the neonatal respiratory distress syndrome (RDS). Since RDS is often complicated by periventricular haemorrhage (PVH) [6], it is important to know to what extent PEEP influences the brain. In the present study this question is restricted to the effect of PEEP on the cerebral blood flow (CBF) which is considered to be a key factor in the pathogenesis of PVH [25].

Shortland et al. [21] have shown that PEEP increases CBF velocity (CBFV) in the anterior cerebral artery and postulated that $\mathrm{P}_{\mathrm{a}} \mathrm{CO}_{2}$ mediates this increase. Others however, reported that PEEP hardly affects $\mathrm{P}_{2} \mathrm{CO}_{2}[4,26]$. Thus, other mechanisms have to be considered.

Another question is whether PEEP affects CBF fluctuation (CBFF). This question which to our knowledge has not yet been investigated, is relevant in view of earlier reports that CBFF is related to mechanical ventilation [10,16] and PVH [11]. This question and the uncertainty regarding the mechanism underlying the effect of PEEP on CBFV, were the motives for the present study.

\section{Patients and methods}

\subsection{Patients}

Study patients were 17 preterm newborns who developed RDS and required assisted ventilation with PEEP. Infants with congenital malformations were excluded. Gestational age was $\leq 35$ weeks. Parental consent was obtained in all cases. Eight infants developed severe complications: five PVH (two of them died); two seizures; and one died for unexplained reasons. In nine infants, the RDS followed an uncomplicated course. The antecedents of the infants are shown in Table 1. None of them required paralysation or developed pneumothorax.

\subsection{Study design}

The present study is part of a larger research project which includes measurements of CBFV and CBFF in the supraclinoidal part of the internal carotid arteries in the

Table 1

Clinical characteristics of the RDS patients

\begin{tabular}{lcc}
\hline & $\begin{array}{c}\text { Uncomplicated } \\
(n=9)\end{array}$ & $\begin{array}{c}\text { Complicated } \\
(n=8)\end{array}$ \\
\hline Gestational age (weeks) & $28-32$ & $26-35$ \\
Birth weight (g) & $815-1900$ & $985-2750$ \\
Weight percentile & $5-75$ & $10-75$ \\
Apgar score after $5 \mathrm{~min}$ & $5-10$ & $6-10$ \\
Male & 4 & 5 \\
Complicated pregnancy & 9 & 6 \\
Born by cesarean section & 7 & 2. \\
\hline
\end{tabular}

The numbers represent ranges $(-)$ or number of infants. 
first 5 days of life by means of Doppler ultrasound. In mechanically ventilated infants, presented in this paper, the measurements were repeated during a period of maximally $5 \mathrm{~min}$, during which PEEP was lowered from a standard 4 to $0 \mathrm{mbar}$. The difference $(\Delta)$ of the PEEP-less measurements minus the PEEP- or baseline measurements served as a measure of the effect of PEEP-interruption. This difference was calculated for six blood flow parameters (including end diastolic, mean and peak systolic CBFV and CBFF) and for heart rate and behaviour.

The measurements were completed with a determination of ductal left to right shunting and a neurological examination. The whole procedure was scheduled within $12 \mathrm{~h}$ after birth and was repeated on the second half of the first day and on the 2 nd, 3rd and 5th day, as long as ventilation took place. It took place only if seizures and bradycardia had been absent for at least $30 \mathrm{~min}$ and was cancelled if it exceeded $20 \mathrm{~min}$. The study protocol was approved by the hospital ethical committee.

\subsection{Blood flow parameters}

The apparatus used was a duplex ultrasound system (manufactured by ATL) consisting of a $5-\mathrm{MHz}$ range gated Doppler velocimeter combined with a real time mechanical sector scanner. Both carotid arteries were transfontanellary insonated, just proximal to their bifurcation. The trapezoidal shape of the chiasmatic cistern and the pulsations of the carotid and middle cerebral arteries, served as reference points. As the carotid arteries are thus still imperfectly visualised, they were scanned with Doppler ultrasound. The position which produced a Doppler signal of maximal frequency and intensity was chosen as the site of measurement. This unequivocally defined position presents a negligible angle of insonation and optimal inclusion of the artery in the sample volume.

According to the study protocol, the baseline measurement was performed in fourfold, i.e. on both sides in duplicate (to estimate repeatability) and the PEEP-less measurement in twofold (one on both sides). Per measurement, one epoch of $20 \mathrm{~s}$ without behavioral state transitions or crying was analyzed pulse by pulse with the ECG as a time reference. In the thus obtained sets of pulse data, the median and interquartile range for the end diastolic, mean and peak systolic velocity were calculated [9]. The median served as a measure of CBFV and the interquartile range as a measure of CBFF.

\subsection{Other parameters}

Heart rate was derived from the ECG and was calculated in the same way as CBFV. Ductal shunting was determined by means of a Doppler recording of the blood flow in the main pulmonary artery [2]. The neurological examination took place according to Volpe [24] and included records of behaviour, perception, posture, muscle tone, body and limb movements, cranial nerves, reflexes and habituation and included also a transfontanellar ultrasound brain scan. For reasons discussed previously [9], behaviour was scored by means of two variables, each with two levels, i.e. wakefulness (eyes open/closed) and restlessness (gross movements or irregular respiration present/absent). The diagnosis PVH was made from the brain 
scan on criteria according to Volpe [24]. In all instances, follow-up imaging and postmortem examination confirmed the initial diagnosis.

\subsection{Statistics}

The effect of PEEP-interruption or $\Delta$ was calculated per artery and averaged for both sides. Left-right differences and the significance of $\Delta$ were tested by means of the Wilcoxon signed rank test. Bias by multiple observations per infant was avoided by selecting one PEEP-procedure per infant. Selection took place by means of the SAS RANUNI random number generator [19]. The influence of age was tested by means of Pearson correlation, applied to all measurements.

Explanatory factors were tested by means of backward stepwise regression analysis applied to the measurements in uncomplicated RDS. Bias by multiple observations per infant was avoided by including patient number (a number assigned to each infant) as a co-variable. Further co-variables were: $\Delta$ restlessness; $\Delta$ wakefulness; $\Delta$ heart rate; PDA; and the baseline of the blood flow parameter in question. Differences between complicated and uncomplicated RDS were analyzed likewise, and only co-variables that appeared significant in uncomplicated RDS were taken into account. The results were checked for normal distribution of the residuals and bias due to regression to the mean. Only $P$-values $<0.05$ were considered significant.

\section{Results}

Fifty-two complete measurement procedures were eligible for further analysis: 22 in the eight infants with complicated RDS and 30 in the nine infants with uncomplicated RDS. Measurements were omitted because of: termination of mechanical ventilation ( $n=15)$; unstable behaviour or crying $(n=10)$; death $(n=6)$; and seizures $(n=2)$. Adverse reactions requiring termination of the measurements did not occur. The baseline conditions before PEEP interruption are shown in Table 2.

Table 2

Baseline conditions of the RDS patients

\begin{tabular}{lcc}
\hline & $\begin{array}{c}\text { Uncomplicated } \\
(n=30)\end{array}$ & $\begin{array}{c}\text { Complicated } \\
(n=22)\end{array}$ \\
\hline End diastolic CBFV (cm/s) & $-2.4-16.2$ & $-2.9-13.0$ \\
Mean CBFV (cm/s) & $3.8-26.0$ & $2.8-22.8$ \\
Peak systolic CBFV (cm/s) & $11.4-36.6$ & $9.3-38.1$ \\
End diastolic CBFF (cm/s) & $1.3-8.3$ & $1.4-4.6$ \\
Mean CBFF (cm/s) & $0.7-3.9$ & $0.7-2.4$ \\
Peak systolic CBFF (cm/s) & $1.2-3.6$ & $0.7-3.1$ \\
Heart rate (beats/min) & $117-176$ & $101-171$ \\
Quiet & 27 & 20 \\
Eyes closed & 24 & 18 \\
Patent ductus arteriosus & 21 & 15 \\
\hline
\end{tabular}

The numbers represent ranges (-) or numbers of observations; CBFV, cerebral blood flow velocity; CBFF, cerebral blood flow fluctuation. 
Table 3

Changes $(\Delta)$ induced by PEEP interruption

\begin{tabular}{lll}
\hline & $\begin{array}{l}\text { Uncomplicated } \\
\text { RDS }(n=30)\end{array}$ & $\begin{array}{l}\text { Complicated RDS } \\
(n=22)\end{array}$ \\
\hline dend diastolic CBFV (cm/s) & $-6.0-1.9^{*}$ & $-5.8-0.5^{*}$ \\
$\Delta$ mean CBFV (cm/s) & $-7.4-1.8^{*}$ & $-7.9-1.1^{* *}$ \\
$\Delta$ peak systolic CBFV (cm/s) & $-9.8-1.9$ & $-7.8-2.9$ \\
$\Delta$ end diastolic CBFF (cm/s) & $-3.2-1.0$ & $-1.4-0.8$ \\
$\Delta$ mean CBFF (cm/s) & $-1.2-0.9$ & $-0.9-0.3$ \\
$\Delta$ peak systolic CBFF (cm/s) & $-1.4-0.9$ & $-1.1-0.8$ \\
Aheart rate (beats/min) & $-7.2-9.3$ & $-5.3-10.1$ \\
$\Delta$ restlessness & 2 & 2 \\
$\Delta$ wakefulness & 8 & 9 \\
\hline
\end{tabular}

The numbers represent ranges (-) or numbers of observations; CBFF, cerebral blood flow velocity; CBFF, cerebral blood flow fluctuation.

$* P<0.05$.

**P $<0.01$.

The raw effects of PEEP-interruption are shown in Table 3. Significant left-right or age differences were not found. Hence, both sides have been taken together and shown irrespective of age. The figures show that PEEP interruption causes a decrease in CBFV, but no consistent changes in CBFF, heart rate or behaviour.

The multiple regression analysis of $\triangle \mathrm{CBFV}$ and $\triangle \mathrm{CBFF}$ is elaborated in Table 4. Most of the co-variables taken into account are involved in $\triangle \mathrm{CBFV}$ or $\triangle \mathrm{CBFF}$, but patient number is by far the most important. The negative regression coefficients for PDA and the baseline of the blood flow parameters imply that the effect of PEEP interruption on CBFV (i.e: a decrease) is more pronounced when the ductus arteriosus is patent or CBFV is high. It further implies that strong CBFF tends to decrease less during PEEP interruption than weak CBFF. The positive coefficient for $\triangle$ restlessness indicates that the end diastolic CBFV decrease is relatively small when PEEP interruption is accompanied by restlessness. Additional analyses revealed no violation of the normal distribution assumption and no significant bias due to regression to the mean. The differences between complicated and uncomplicated RDS are presented in Table 5. These were not significant. Reduction of the number of co-variables did not substantially change this.

\section{Discussion}

Doppler technology allows measurement of CBFV and CBFF, but not of the volume of CBF. Further, the internal carotid artery is not the sole artery that supplies the brain. It is however generally accepted that Doppler ultrasound applied to the major cerebropetal arteries provides a valuable estimate of CBF [23]. This holds a fortiori for the changes induced by interventions as presented in this study. The interquartile range was used as a measure of fluctuation, as it is more specific for fluctuation than the coefficient of variation [9]. 


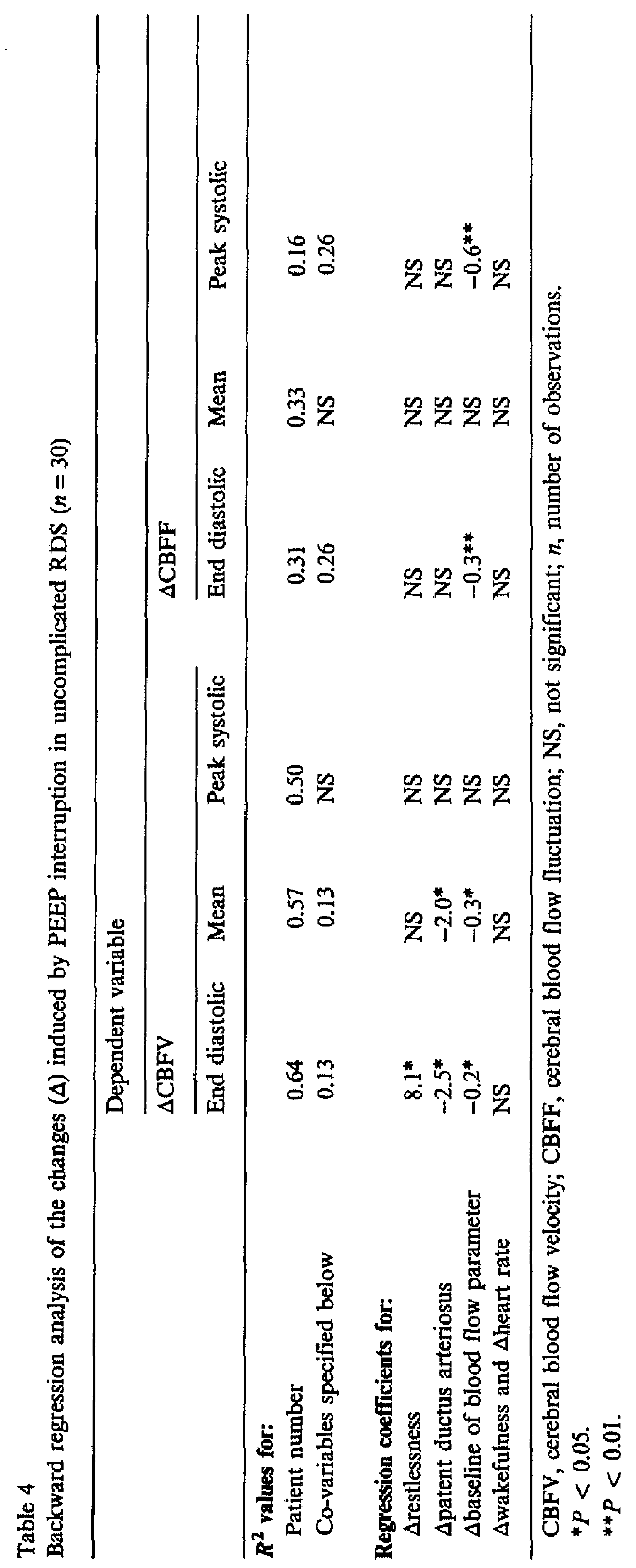


Table 5

Differences (cm/s \pm S.E.) between complicated RDS $(n=22)$ and uncomplicated RDS $(n=30)$ for the changes $(\Delta)$ induced by PEEP interruption

\begin{tabular}{|c|c|c|c|c|c|}
\hline \multicolumn{3}{|l|}{$\triangle \mathrm{CBFV}$} & \multicolumn{3}{|l|}{$\triangle \mathrm{CBFF}$} \\
\hline End diastolic & Mean & Peak systolic & End diastolic & Mean & Peak systolic \\
\hline$-0.7 \pm 0.4$ & $-0.7 \pm 0.5$ & $-0.2 \pm 0.6$ & $-0.1 \pm 0.2$ & $-0.2 \pm 0.1$ & $-0.2 \pm 0.2$ \\
\hline
\end{tabular}

Calculated by fitting a linear regression model including the following co-variables: patient number; $\Delta$ restlessness; patent ductus arteriosus; and baseline of the blood flow parameter in question. None of the differences are significant; $n$, number of observations; CBFV, cerebral blood flow velocity; CBFF, cerebral blood flow fluctuation.

Our finding that PEEP interruption decreases CBFV, is in agreement with an earlier observation of Shortland et al., that PEEP elevation increases mean CBFV in the anterior cerebral artery [21]. Hence the effect of PEEP on CBF is reversible and immediate, and affects all major cerebral arteries. According to our findings, this effect applies especially to the end diastolic CBFV and not at all to CBFF.

Various mechanisms can be held responsible for the decrease in CBFV during PEEP interruption. The $R^{2}$ values in Table 4, in particular those for patient number, imply that other factors are still more important than those explicitly taken into account. Perhaps, the intracranial pressure is such a factor [20].

The fact that end diastolic CBFV decreases less when an infant becomes restless, might imply that restlessness counteracts the cerebral hypoxia intrinsic to the decrease in CBF during PEEP interruption. This assumption is consistent with reports that cerebral hypoxia causes arousal [3] and that restlessness increases CBF [14].

Heart rate and shunting across the ductus arteriosus were studied in view of the fact that PEEP affects left ventricular function $[5,12]$ and ductal shunting $[1,12]$ and thus $\mathrm{CBF}$ as well. Shunting across PDA decreases CBF [7]. PEEP counteracts ductal shunting [1] and will therewith reduce the negative effect of PDA on CBF. This explains our finding that PDA accentuates the effect of PEEP interruption on end diastolic and mean CBFV.

The baselines of the blood flow parameters were taken into account as they reflect dynamics of $\mathrm{CBF}$ and therewith its stability. Furthermore, PEEP interruption might affect CBF in a proportional way, just as holds for many other biological interventions. Accordingly, a high CBFV might be more susceptible to ductal shunting and hence might be more dependent on the counteracting effect of PEEP on ductal shunting. This might explain our finding that a negative relationship exists between the CBFV baseline and the effect of PEEP interruption, or in other words that a high CBFV decreases more than on a low CBFV. The similar finding for CBFF is hard to explain. In view of the beneficial effects of PEEP on the systemic circulation $[1,12]$ and on respiratory effort [12,22], one would expect a positive relationship.

Complicated and uncomplicated RDS were studied separately, since a poor condition might affect $C B F$ and hence $\triangle \mathrm{CBF}$. Brain damage which largely accounts for 
the differences between our two groups of RDS patients, disturbs CBF regulation $[13,15,17]$. This disturbance might impair the capability to react to the strain of PEEP interruption. This coincides with our finding that CBFV decreases more in complicated than in uncomplicated RDS. Medication has been left out of consideration, since its role seems to be small [18].

\section{Conclusions and speculations}

It is concluded that PEEP supports CBF in preterm newborns with RDS by decreasing ductal stealing from the brain. This makes PEEP a measure which is beneficial to the brain, as cerebral hypoperfusion is a common denominator of neonatal brain damage and RDS. Our findings further indicate that the decrease in CBF which occurs during PEEP interruption is moderated by restlessness and accentuated by brain damage. The outlined procedure thus not only provides an insight into the influence of PEEP on CBF, but might also serve as a test for the integrity of $\mathrm{CBF}$. In view of the preliminary character of our findings this needs to be further established.

\section{References}

[1] Björkhem, G.E., Lundström, N.-R. and Svenningsen, N.W. (1977): Influence of continuous positive pressure treatment on ductus arteriosus shunt assessed by echocardiography. Arch. Dis. Child., 52, 658-661.

[2] Daniels, O., Hopman, J.C.W., Stoelinga, G.B.A., Busch, H.J. and Peer, P.G.M. (1982): Doppler flow characteristics in the main pulmonary artery and the LA/Ao ratio before and after ductal closure in healthy newborns. Pediatr. Cardiol., 3, 99-104.

[3] Fewell, J.E. and Baker, S.B. (1987): Arousal from sleep during rapidly developing hypoxemia in lambs. Pediatr. Res., 22, 471-477.

[4] Greenough, A., Chan, V. and Hird, M.F. (1992): Positive end expiratory pressure in acute and chronic respiratory distress. Arch. Dis. Child., 67, 320-323.

[5] Jardin, F., Farcot, J., Boisante, L., Curien, N., Margairaz, A. and Bourdarias, J. (1981); Influence of positive end-expiratory pressure on left ventricular performance. N. Engl. J. Med., 304, 387-392.

[6] Leviton, A., Van Marter, L. and Kuban, K.C.K. (1989): Respiratory distress syndrome and intracranial haemorrhage: cause or association? Inferences from surfactant clinical trials. Pediatrics, 84 , 915-922.

[7] Mellander, M, and Larsson, L.E. (1988): Effects of left-to-right ductus shunting on left ventricular output and cerebral blood flow velocity in 3-day-old preterm infants with and without severe lung disease. J. Pediatr., 113, 101-109.

[8] Mullaart, R.A., Hopman, J.C.W., Daniels, O., Rotteveel, J.J., De Haan, A.F.J. and Vreuls, H.J.M. (1989): Repeatability of Doppler ultrasound measurement of blood flow velocity and its variability in the supraclinoid segment of the internal carotid artery in preterm newborns. Ultrasound Med. Biol. 15, 545-553.

[9] Mullaart, R.A., Hopman, J.C.W., De Haan, A.F.J., Rotteveel, J.J., Daniels, O. and Stoelinga, G.B.A. (1992): Cerebral blood flow fluctuation in low-risk preterm newborns. Early Hum. Dev., $30,41-48$.

[10] Perlman, J.M., McMenamin, J.B. and Volpe, J.J. (1983): Fluctuating cerebral blood-flow velocity in respiratory-distress syndrome. N. Engl. J. Med., 309, 204-209.

[11] Perlman, J.M., Goodman, S., Kreusser, K.L. and Volpe, J.J. (1985): Reduction of intraventricular haemorrhage by elimination of fluctuating cerebral blood-flow velocity in preterm infants with respiratory distress syndrome. N. Engl. J. Med., 312, 1353-1357. 
[12] Polin, R.A. and Fox, W.W. (1992): Fetal and Neonatal Physiology, pp. 892-893. Saunders, Philadelphia, PA.

[13] Pryds, O. (1991): Control of cerebral circulation in the high-risk neonate. Ann. Neurol., 30, 321-329.

[14] Ramaekers, V.Th., Casaer, P., Daniels, H., Smet, M. and Marchal, G. (1989): The influence of behavioral states on cerebral blood flow velocity patterns in stable preterm infants. Early Hum. Dev., $20,229-246$.

[15] Ramaekers, V.Th. and Casaer, P. (1990): Defective regulation of cerebral oxygen transport after severe birth asphyxia. Dev. Med. Child Neurol., 32, 556-562.

[16] Rennie, J.M., South, M. and Morley, C.J. (1987): Cerebral blood flow velocity variability in infants receiving assisted ventilation. Arch. Dis. Child., 62, 1247-1251.

[17] Rosenberg, A.A. (1988): Regulation of cerebral blood flow after asphyxia in neonatal lambs. Stroke, 19, 239-244.

[18] Saliba, E., Autret, E., Nasr, C., Suc, A.L. and Laugier, J. (1992): Perinatal pharmacology and cerebral blood flow. Biol. Neonate, 62, 252-257.

[19] SAS Inst. Inc. (1985): SAS ${ }^{\oplus}$ Users Guide: Statistics, version 5th edition. SAS Inst. Inc., Cary, NC.

[20] Shapiro, H.M. and Marshal, L.F. (1978): Intracranial pressure responses to PEEP in head-injured patients. J. Trauma, 18, 254-256.

[21] Shortland, D.B., Field, D., Archer, L.N.J., Gibson, N.A. et al. (1989): Cerebral hemodynamic effects of changes in positive end expiratory pressure in preterm infants. Arch. Dis. Child., 64, 465-469.

[22] Speidel, B.D. and Dunn, P.M. (1975): Effect of continuous positive airway pressure on breathing pattern of infants with respiratory distress syndrome. Lancet, 8 February, 302-304.

[23] Taylor, G.A., Short, B.L., Walker, L.K. and Traystman, R.J. (1990): Intracranial blood flow: quantification with duplex Doppler and colour Doppler flow US. Radiology, 176, 231-236.

[24] Volpe, J.J. (1987): Neurology of the Newborn, pp. 70-96. Saunders, Philadelphia, PA.

[25] Volpe, J.J. (1989): Intraventricular haemorrhage in the premature infant - current concepts. Part I. Ann. Neurol., 25, 3-11.

[26] Yu, V.Y.H. and Rolfe, P. (1977): Effect of continuous positive airway pressure breathing on cardiorespiratory function in infants with respiratory distress syndrome. Acta Paediatr. Scand., 66, $59-64$. 